\title{
A proteomics-based approach identifies secreted protein acidic and rich in cysteine as a prognostic biomarker in malignant pleural mesothelioma
}

\begin{abstract}
Steven C Kao*,1,2,3, Michaela B Kirschner ${ }^{1,12}$, Wendy A Cooper ${ }^{3,4,5}$, Thang Tran ${ }^{4}$, Sjaak Burgers ${ }^{6}$, Casey Wright ${ }^{1}$, Tiny Korse ${ }^{6}$, Daan van den Broek ${ }^{6}$, James Edelman ${ }^{7}$, Michael Vallely ${ }^{3,7,8}$, Brian McCaughan ${ }^{3,9}$, Nick Pavlakis ${ }^{3,10}$, Stephen Clarke ${ }^{3,10}$, Mark P Molloy ${ }^{11}$, Nico van Zandwijk ${ }^{1,3}$ and Glen Reid ${ }^{1,3}$

${ }^{1}$ Asbestos Diseases Research Institute, PO Box 3628, Rhodes, Sydney, NSW2139, Australia; ${ }^{2}$ Department of Medical Oncology, Chris O'Brien Lifehouse, Sydney, NSW 2050, Australia; ${ }^{3}$ Sydney Medical School, The University of Sydney, Sydney, NSW 2006, Australia; ${ }^{4}$ Department of Tissue Pathology and Diagnostic Oncology, Royal Prince Alfred Hospital, Sydney, NSW 2050, Australia; ${ }^{5}$ University of Western Sydney, Sydney, NSW 2150, Australia; ${ }^{6}$ Division of Thoracic Oncology, The Netherlands Cancer Institute, Amsterdam 1066 CX, The Netherlands; ' Department of Cardiothoracic Surgery, Royal Prince Alfred Hospital, Sydney, NSW 2050, Australia; ${ }^{8}$ Australian School of Advanced Medicine, Macquarie University, Sydney, NSW 2109, Australia; ${ }^{9}$ Sydney Cardiothoracic Surgeons, RPAH Medical Centre, Sydney, NSW 2050, Australia; ${ }^{10}$ Department of Medical Oncology, Royal North Shore Hospital, Sydney, NSW 2065, Australia and ${ }^{11}$ Australian Proteome Analysis Facility, Macquarie University, Sydney, NSW 2109, Australia
\end{abstract}

Background: We aimed to identify prognostic blood biomarkers using proteomics-based approaches in malignant pleural mesothelioma (MPM).

Methods: Plasma samples from 12 MPM patients were used for exploratory mass spectrometry and ELISA analyses. The significance of secreted protein acidic and rich in cysteine (SPARC) was examined in sera from a Dutch series $(n=97)$. To determine the source of the circulating SPARC, we investigated SPARC expression in MPM tumours and healthy controls, as well as the expression and secretion from cell lines and xenografts.

Results: Secreted protein acidic and rich in cysteine was identified as a putative prognostic marker in plasma. Validation in the Dutch series showed that the median survival was higher in patients with low SPARC compared with those with high SPARC (19.0 vs 8.8 months; $P=0.01$ ). In multivariate analyses, serum SPARC remained as an independent predictor (HR 1.55; $P=0.05)$. In MPM tumour samples, SPARC was present in the tumour cells and stromal fibroblasts. Cellular SPARC expression was higher in 5 out of 7 cell lines compared with two immortalized mesothelial lines. Neither cell lines nor xenograft tumours secreted detectable SPARC.

Conclusions: Low circulating SPARC was associated with favourable prognosis. Secreted protein acidic and rich in cysteine was present in both tumour cells and stromal fibroblasts; and our in vitro and in vivo experiments suggest that stromal fibroblasts are a potential source of circulating SPARC.

${ }^{*}$ Correspondence: Dr SC Kao; E-mail: steven.kao@lh.org.au
${ }^{12}$ Current address: Division of Thoracic Surgery, University Hospital Zurich, 8091 Zurich, Switzerland.

Received 27 August 2015; revised 26 October 2015; accepted 19 November 2015; published online 18 February 2016

(C) 2016 Cancer Research UK. All rights reserved 0007-0920/16 
Malignant pleural mesothelioma (MPM) is an almost invariably fatal, asbestos-related malignancy arising from the mesothelium surrounding the thoracic cavities. Progress in MPM treatment has remained modest, with a median survival varying from 7 months to 2 years (Vogelzang, 2008; Van Meerbeeck et al, 2011). Use of the few treatment options available is guided by traditional prognostic factors such as performance status, histological subtype and stage of disease (Scherpereel et al, 2010; Van Meerbeeck et al, 2011). The use of pemetrexed/cisplatin chemotherapy is associated with a response rate of around $40 \%$ and a survival gain of around 3 months (Vogelzang et al, 2003). None of the molecular markers investigated to date is able to accurately predict the course of the disease or the response to therapy. A biomarker, or a combination of biomarkers, that is able to determine prognosis or response to therapy and which is accurate enough to be used for the selection of individual patients is therefore urgently needed. Ideally, a simple blood-based biomarker would be advantageous given its easy accessibility.

There are several blood-based biomarkers that have been shown to have some diagnostic utilities. These include osteopontin, soluble mesothelin-related peptides (SMRP) and more recently, fibulin-3 (Grigoriu et al, 2007; Roe et al, 2008; Schneider et al, 2008; Pass et al, 2012). These diagnostic biomarkers also have a limited prognostic role in MPM, with elevated levels of osteopontin and SMRP showing correlation with shorter survival (Grigoriu et al, 2007; Roe et al, 2008; Schneider et al, 2008). Although the concentration of fibulin-3 in the plasma did not correlate with survival, high levels in effusions were associated with shorter survival (Pass et al, 2012).

Identification of circulating blood-based biomarkers has also been attempted via proteomics-based approaches. Ostroff et al (2012) discovered and validated a panel of serum biomarkers as a non-invasive proteomics-based surveillance tool in the early diagnosis of MPM in patients at high risk for this disease. Using the Selected Reaction Monitoring (SRM) assay technology, Cerciello et al (2013) also identified serum protein-derived candidate biomarker panels for the diagnosis of MPM.

Given the feasibility of biomarker identification using proteomics-based technology and the lack of accurate prognostic biomarkers in MPM, we initially designed an exploratory proteomic study, in which we systematically investigated proteins present in plasma of MPM patients using the isobaric tag for relative and absolute quantification (iTRAQ) mass spectrometry (Ross et al, 2004). This identified secreted protein acidic and rich in cysteine (SPARC; or osteonectin) as a promising prognostic biomarker. Further investigation suggested that circulating levels of SPARC appear to originate from the stroma.

\section{MATERIALS AND METHODS}

Patients and clinical materials. Three retrospective series of MPM patients were included in this study. The discovery series consisted of 12 patients from the parallel, non-randomized phase II studies investigating the effectiveness of thalidomide (arm A: thalidomide in combination with cisplatin and gemcitabine $[n=34]$; and arm B: thalidomide alone $[n=29]$, recruited between April 2001 and August 2003) (Kao et al, 2012) consisting of the six shortest and six longest surviving patients (median overall survival [OS] 1.2 and 38.3 months, respectively; Supplementary Table 1 for patient characteristics). This sample set was used to identify circulating biomarkers associated with OS differences. Overall survival was defined as the time from enrolment to death, while progression-free survival (PFS) was defined as the time from enrolment to progression (or death if progression had not occurred). Collected blood was centrifuged at $2500 \mathrm{~g}$ for $10 \mathrm{~min}$, and separated plasma was stored at $-80^{\circ} \mathrm{C}$. Patients gave written informed consent under the protocol approved by the Human Research Ethics Committee (HREC) at Royal North Shore Hospital.

The second series consisted of 97 pathologically confirmed MPM patients treated at the Netherlands Cancer Institute between 1995 and 2011. Patients donated blood samples at the time of diagnosis, which were spun at 3100 r.p.m. for $10 \mathrm{~min}$ to isolate serum, which was then stored at $-30^{\circ} \mathrm{C}$. This series was used for validation of candidate circulating protein biomarkers, which is the primary analysis in this study. Overall survival was defined as the time from diagnosis to death. Patients gave consent under the Institutional Biobank Programme approved by HREC at the Antoni van Leeuwenhoek Hospital-Netherlands Cancer Institute.

The third series consisted of archival tumour samples from 74 MPM patients treated with palliative pleurectomy \pm decortication (P/D) at Royal Prince Alfred Hospital (RPAH), Sydney between 1991 and 2009. Overall survival in this series was defined as the time from the surgical procedure to death. These formalin-fixed paraffin-embedded (FFPE) samples were used to examine tumour SPARC expression. Waiver of consent was approved by HREC at Sydney Local Health District (SLHD) due to the retrospective nature of the series.

Normal mesothelium $(n=13)$ was used as a control with the third series and consisted of patients without a history of malignancy or inflammatory conditions who underwent cardiac or aortic surgery at RPAH. Patients gave written informed consent, and the collection of normal mesothelium was approved by HREC at SLHD.

iTRAQ. Patient plasma samples from the first series were immunodepleted of the top 14 most abundant plasma proteins using a multiple affinity removal system (Agilent Technologies, Santa Clara, CA, USA) following the manufacturer's direction. The unbound fraction was collected and precipitated in ice-cold acetone overnight. The sample was centrifuged at $14000 \mathrm{~g}$ for $10 \mathrm{~min}$ to pellet the proteins. The acetone was decanted and pellet resuspended with $0.1 \mathrm{M}$ triethylammonium bicarbonate before labelling for iTRAQ analysis. A common reference sample consisting of a pool of all samples was used in each iTRAQ experiment as the denominator for determining ratios. Samples were labelled by following the manufacturer's protocol with modifications as previously described (Song et al, 2008). Tryptic peptides were fractionated by strong cation exchange chromatography then each fraction analysed by information-dependent acquisition using reversedphase nanoLC/MS using a TripleTOF 5600 mass spectrometer (ABSciex, Framingham, MA, USA). Data files were searched against the Human SWISS-PROT protein database using ProteinPilot (v4.0, ABSciex) with thorough search mode and bias correction. Proteins were assigned with a false discovery rate of $<1 \%$. Differential expression of proteins measured by iTRAQ between short- and long-MPM survivors was assessed using an empirical Bayes moderated $t$-test as implemented in the limma library from Bioconductor (Gentleman et al, 2004), as we have previously described (Jankova et al, 2011). A test $P$-value of $\leqslant 0.05$ was regarded as evidence of differential abundance.

ELISA. The protein candidates were validated by quantification using commercially available ELISA kits in accordance with the manufacturers' instruction, carried out by researchers blinded to the study endpoint. These included Selenoprotein P (qy-e01007; Qayee-Bio for Life Science, Shanghai, China), Tetranectin (E80350Hu; Uscn Life Science Inc, Wuhan, China) and SPARC (E90791Hu; Uscn Life Science Inc). Duplicate measures were made for each sample.

Protein levels were calculated and expressed as either $\mu \mathrm{g} \mathrm{ml}^{-1}$ (Selenoprotein P, Tetranectin and SPARC in plasma and serum) or ng per total protein (SPARC in cell lysates and cell-conditioned medium). 
Tissue microarrays. Formalin-fixed paraffin-embedded tumour tissue blocks from the P/D procedure and normal mesothelium blocks from cardiac surgery were retrieved. Areas containing tumour and normal mesothelium, respectively, were marked on the haemotoxylin and eosin slides; and the corresponding areas were determined on the FFPE blocks. Tissue microarrays (TMAs) were constructed as described previously (Kao et al, 2011). Briefly, a minimum of four 1-mm cores were sampled from the FFPE blocks and inserted into a recipient block, using the ATA-100 Advanced Tissue Arrayer (Chemicon, Temecula, CA, USA). Cores of smooth muscle were included as negative controls in each TMA (Supplementary Figure 1).

Immunohistochemistry. Serial $4-\mu \mathrm{m}$ paraffin sections of the TMA blocks underwent immunohistochemical staining for SPARC. Sections were incubated with primary rabbit polyclonal anti-SPARC antibody (AB14174, dilution 1: 1000; Abcam, Boston, MA, USA) for $32 \mathrm{~min}$ at room temperature after using CC2 (Ventana Medical Systems, Tucson, AZ, USA) as an antigen retrieval solution (citrate buffer $\mathrm{pH}$ 6) for $44 \mathrm{~min}$ at $97^{\circ} \mathrm{C}$. The reaction was developed with the UltraView DAB detection kit (Ventana Medical Systems), using BenchMark ULTRA (Ventana Medical Systems).

Immunostaining evaluation. Immunostaining was assessed by an expert pulmonary pathologist (WAC) who was blinded to the clinical data. Positive labelling was defined as cells with cytoplasmic staining of any intensity. The percentage of cells labelled by the antibodies $(0-100 \%)$ was recorded in all assessable cores in a semiquantitative manner. Separate scores were determined for both the tumour cells and the stromal fibroblasts. The final score for SPARC staining in samples from each patient was calculated from the average score of the available cores in the TMAs.

Cell lines: cell-conditioned medium and protein lysates. Five human mesothelioma cell lines (H28, H226, H2452 and MSTO) and the immortalized mesothelial cell line MeT-5A were obtained from the American Type Culture Collection (Manassas, VA, USA; it should be noted here that although listed as mesothelioma by ATCC, it has been debated whether the origin of the NCI-H226 cell line was a mesothelioma or a pleural metastasis from a squamous cell carcinoma of the lung). In addition, the previously described MPM lines MM05 (Relan et al, 2013), VMC23 (Kryeziu et al, 2013) and SPC111 (Schmitter et al, 1992) and the mesothelial line LP9 (Wu et al, 1982) were included. Cell lines were grown in their corresponding medium (RPMI-1640 for all MPM lines, DMEM for MeT-5A, M199 supplemented with 3.3 nM EGF, 400 nM hydrocortisone and $1 \%$ insulin-transferrin-selenium for LP9) supplemented with $10 \%$ fetal bovine serum (FBS) at $37^{\circ} \mathrm{C}, 5 \%$ $\mathrm{CO}_{2}$ and $95 \%$ humidity. All media and supplements were purchased from Life Technologies (Carlsbad, CA, USA) or Sigma-Aldrich (St Louis, MO, USA).

To obtain cell-conditioned medium, cells were grown to $80 \%$ confluence in T25 flasks in medium containing FBS, before medium was replaced with $2 \mathrm{ml}$ serum-free medium. Cellconditioned medium was collected after $24 \mathrm{~h}$, cell debris removed by $5 \mathrm{~min}$ centrifugation at 1200 r.p.m., and supernatants stored at $-80^{\circ} \mathrm{C}$. Protein lysates were prepared from the same cells by lysis in $300 \mu \mathrm{l}$ RIPA buffer as described previously (Linton et al, 2014). Protein concentration was determined using the Pierce BCA Protein Assay Kit (Thermo Scientific, Waltham, MA, USA) according to the manufacturer's instructions.

Mouse plasma and serum samples. Athymic $(n u / n u)$ mice were purchased from the Animal Resources Centre (Perth, WA, Australia) and injected subcutaneously in the left flank with $5 \times 10^{6} \mathrm{H} 226$ or $3 \times 10^{6}$ MSTO cells in $100 \mu \mathrm{l}$ serum-free medium/ growth factor-reduced matrigel mix $(1: 1$, matrigel from $\mathrm{BD}$ Biosciences, Franklin Lakes, NJ, USA). Once tumours reached around $300 \mathrm{~mm}^{3}$, mice were killed and blood was collected via cardiac puncture into either empty or EDTA-containing tubes.
Serum samples were allowed to clot for $60 \mathrm{~min}$ at room temperature before being further processed as for plasma samples (centrifugation at 2500 r.p.m. for $20 \mathrm{~min}$ ), and both were stored at $-80^{\circ} \mathrm{C}$. Animal studies were approved by the SLHD Animal Welfare Committee.

Statistical analysis. The primary end point of the study was OS, with definitions stated above. The primary analysis was the validation of circulating SPARC in the Dutch series. Validation of ELISA protein quantification in plasma and serum was performed using the median protein level as a cutoff, to define the two groups of patients (protein-high $v s$ protein-low). This was defined a priori.

The comparison of the baseline patient characteristics and survival in all patient cohorts was made using IBM SPSS Statistics, Version 19 (SPSS Inc, Chicago, IL, USA): chi-square test for categorical variables; Log-rank test for Kaplan-Meier survival analysis; and Mann-Whitney $U$-test for non-parametric distribution comparison. For the primary analysis of determination of prognostic value of circulating SPARC, multivariate analysis was performed using the Cox proportional hazards regression model incorporating age, gender, stage, histological subtype and circulating SPARC level (SPARC-high vs SPARC-low).

To determine the association of SPARC protein expression in the tumour samples with the clinical characteristics (such as age, gender and histological subtype), we used chi-squared test. Significant variables in the univariate analyses were entered into a logistic regression model without selection; and the results of the logistic regression modelling are presented as odds ratios (ORs) and associated 95\% confidence interval (CIs). A $P$-value of $\leqslant 0.05$ was considered as statistically significant.

\section{RESULTS}

Circulating SPARC has prognostic value in MPM. The 12 patients from series one were used for exploratory proteomic analyses. The total number of proteins identified was 226. The iTRAQ analysis of the samples from the short and long survivors revealed five plasma proteins that showed a statistically significant difference $(P<0.05)$ in abundance. Selenoprotein $\mathrm{P}$ and tetranectin were elevated in longterm survivors, while insulin-like growth factor-binding protein 2 (IBP2), platelet basic protein (CXCL7) and SPARC were found to be reduced in plasma of long-term survivors (Supplementary Table 2). Further quantification by ELISA was performed for selenoprotein P, tetranectin and SPARC (Supplementary Table 3). Median protein levels were used to divide the 12 patients into protein-high $v$ s proteinlow for the individual proteins examined. Using the Kaplan-Meier method, SPARC was the only protein that differentiated the patient OS. Low level of SPARC was associated with long survival (median OS: 32.1 vs 1.2 months for low $v$ high SPARC; $P=0.006$ ). Box plots are shown in Supplementary Figure 2 to demonstrate the differential abundance of SPARC.

To validate the association between SPARC and OS, SPARC levels were measured in samples from 97 patients from series two (patient characteristics are described in Table 1). At the time of the report, 92 patients were deceased. The median age of the patients was 60 years (range: $42-76$ years), and the median OS was 13.5 months (95\% confidence interval [CI]: 9.3-17.8 months). The median SPARC concentration measured by ELISA was $1.75 \mu \mathrm{g} \mathrm{ml}^{-1}$ (range: $0.55-2.76 \mu \mathrm{g} \mathrm{ml}^{-1}$; standard deviation [s.d.] $0.41 \mu \mathrm{g} \mathrm{ml}^{-1}$ ) in this series and was used to categorise patients into SPARC-high and SPARC-low groups. The median OS was significantly higher in patients with SPARC-low (19.0 months; 95\% CI: 12.1-25.9 months) compared with SPARC-high patients (8.8 months; 95\% CI: 7.8-9.9 months, $P=0.01$; Figure 1A). The median OS was higher in female (14.7 months; 95\% CI: 0-30.7) compared with male gender (10.7 months; 95\% CI: 6.6-14.8 
months; $P=0.03$; Figure $1 \mathrm{~B})$. In contrast, OS did not differ significantly for age (median OS: 13.1 vs 13.0 for age $<60$ years and $\geqslant 60$ years respectively; $P=0.60$ ), histological subtype (median OS: 13.7 vs 8.8 months for epithelial and non-epithelial histological subtype; $P=0.15$ ) and stage (median OS: 17.0 vs 10.1 months for stages I-II and III-IV respectively; $P=0.14$ ). Using the multivariate Cox proportional hazard Regression model, incorporating all the above factors, SPARC remained an independent prognostic factor, with low SPARC level being associated with longer survival (HR: $1.55 ; P=0.05$; Table 2).

SPARC is overexpressed in MPM tumours. To determine the potential source of circulating SPARC, we first examined the SPARC protein expression in TMAs generated from archival MPM tumour samples from 74 patients who underwent P/D (series three). Owing to attrition of assessable cores, 72 patients were analysed for tumoral SPARC expression while 69 patients were analysed for stromal fibroblast expression, with 13 samples from normal pleural mesothelium from patients undergoing cardiac or aortic surgery used as controls. Cytoplasmic labelling of SPARC was found in all histological subtypes of MPM with staining in both the tumour cells and the stromal fibroblasts (Figure 2A and B).

Table 1. Baseline characteristics for the series of patients from the Netherlands (series two, $n=97$ )

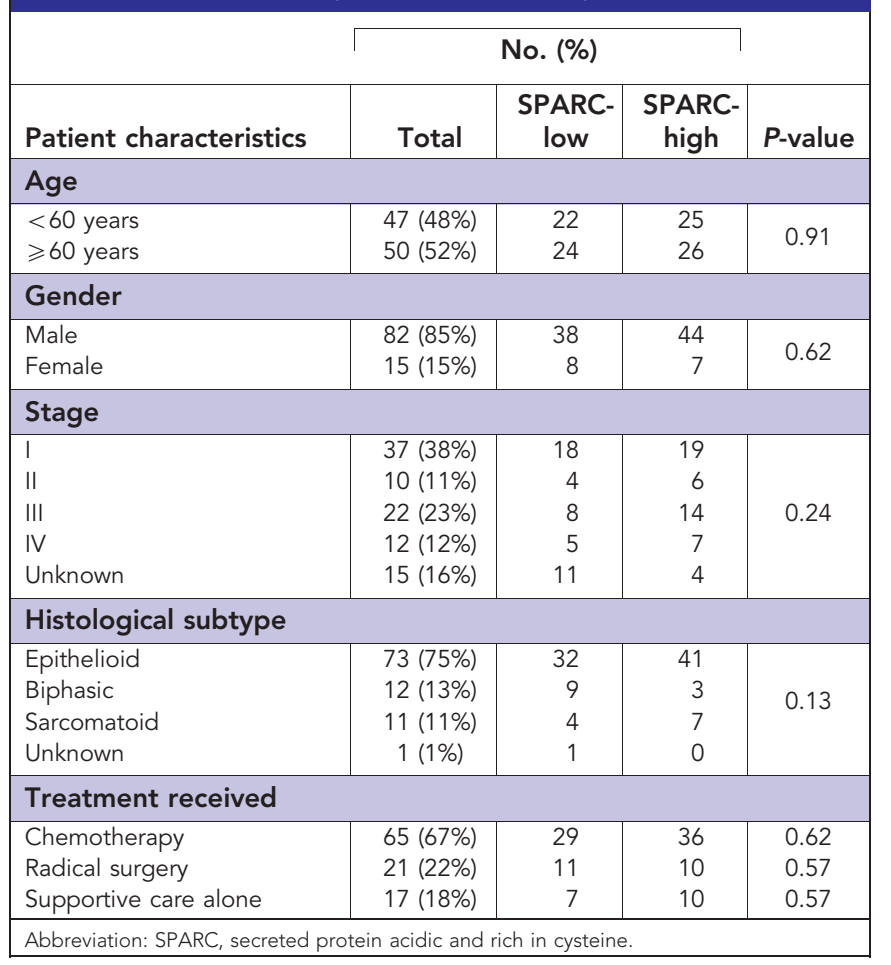

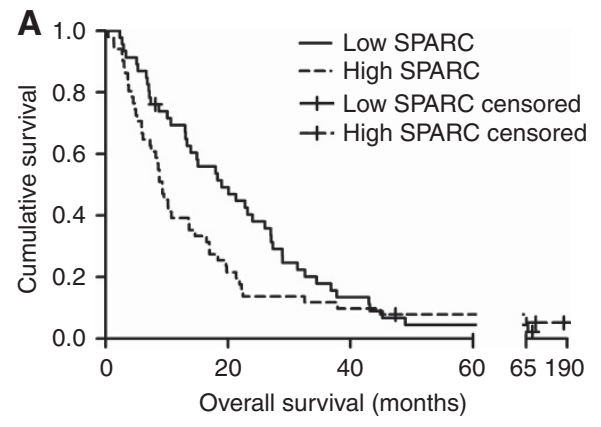

We found both the SPARC IHC scores to be significantly higher in MPM tumour cells $(P=0.001)$ and MPM stromal fibroblasts $(P<0.001)$ compared with respective scores in the normal mesothelium (Figure $2 \mathrm{C}$ and $\mathrm{D}$ ). The SPARC protein in the tumour cells tended to be universally expressed (median expression $100 \%$; range $0-100 \%$ ), while SPARC in the stromal fibroblasts was more heterogeneous (median expression 50\%; range 0-100\%). The box plots in Figure $2 \mathrm{C}$ and $\mathrm{D}$ demonstrate the distribution of the SPARC expression in these $\mathrm{P} / \mathrm{D}$ patients.

Table 3 illustrates that stromal expression of SPARC protein was associated with non-epithelial subtype of MPM. Using logistic regression, high stromal SPARC expression (>50\%) remained independently associated with non-epithelial subtype (OR: 7.7; 95\% CI: $2.3-26.2 ; P=0.001)$. In contrast, SPARC protein tumoral expression was not associated with histological subtype $(P=1.00)$ but female patients were significantly more likely to have SPARC expression in the tumour cells $(P=0.02$; Table 3$)$. In addition, low stromal SPARC $(\leqslant 50 \%)$ was associated with longer survival (10.9 vs 7.6 months; $P=0.001$; Figure $2 \mathrm{E}$ ) while there was no association between tumoral SPARC and patient survival $(P=0.71)$. In contrast, in a multivariate Cox proportional hazard regression model $(n=69)$ incorporating stromal SPARC, age, gender and histological subtype, histological subtype remained an independent predictor of prognosis (HR: 2.47; 95\% CI: 1.38-4.44; $P=0.002$ ), while stromal SPARC did not (HR: 1.42; 95\% CI: 0.76-2.63; $P=0.27$ ). This is likely to be a result of the strong association between histological subtype and stromal SPARC expression.

Table 2. Multivariate analysis of association of baseline prognostic factors with overall survival in patients from the Netherlands (series two; $n=97$ )

\begin{tabular}{|c|c|c|c|}
\hline Factor & HR & $95 \% \mathrm{Cl}$ & $P$-value \\
\hline \multicolumn{4}{|l|}{ Age } \\
\hline \multirow{2}{*}{$\begin{array}{l}<60 \text { years } \\
\geqslant 60 \text { years }\end{array}$} & \multicolumn{2}{|c|}{ Reference } & \multirow{2}{*}{0.346} \\
\hline & 1.25 & $0.79-1.99$ & \\
\hline \multicolumn{4}{|l|}{ Gender } \\
\hline \multirow{2}{*}{$\begin{array}{l}\text { Female } \\
\text { Male }\end{array}$} & \multicolumn{2}{|c|}{ Reference } & \multirow{2}{*}{0.049} \\
\hline & 1.85 & $1.00-3.40$ & \\
\hline \multicolumn{4}{|l|}{ Stage } \\
\hline \multirow{2}{*}{$\begin{array}{l}\text { I-II } \\
\text { III-IV }\end{array}$} & \multicolumn{2}{|c|}{ Reference } & \multirow{2}{*}{0.047} \\
\hline & 1.61 & $1.00-2.58$ & \\
\hline \multicolumn{4}{|c|}{ Histological subtype } \\
\hline \multirow{2}{*}{$\begin{array}{l}\text { Epithelioid } \\
\text { Non-epithelioid }\end{array}$} & \multicolumn{2}{|c|}{ Reference } & \multirow{2}{*}{0.238} \\
\hline & 1.40 & $0.80-2.45$ & \\
\hline \multicolumn{4}{|l|}{ SPARC } \\
\hline \multirow{2}{*}{$\begin{array}{l}\text { Low } \\
\text { High }\end{array}$} & \multicolumn{2}{|c|}{ Reference } & \multirow{2}{*}{0.050} \\
\hline & 1.55 & $1.00-2.42$ & \\
\hline
\end{tabular}

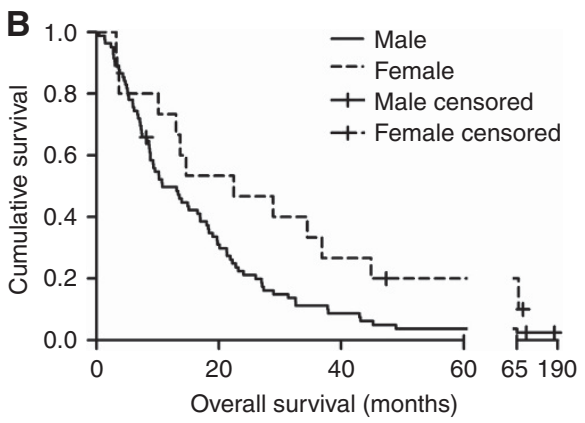

Figure 1. Kaplan-Meier curves for the patients in series two according to serum SPARC (A) and gender (B). 
A

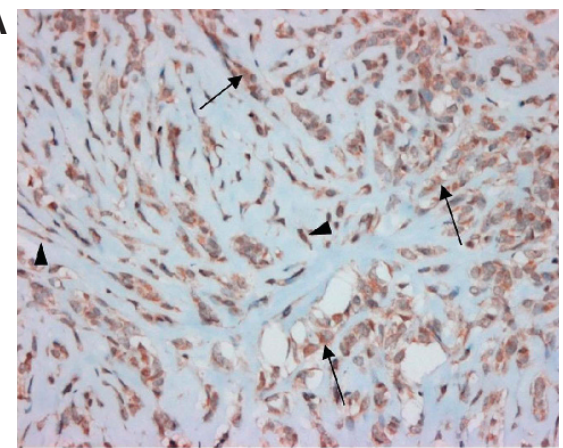

B

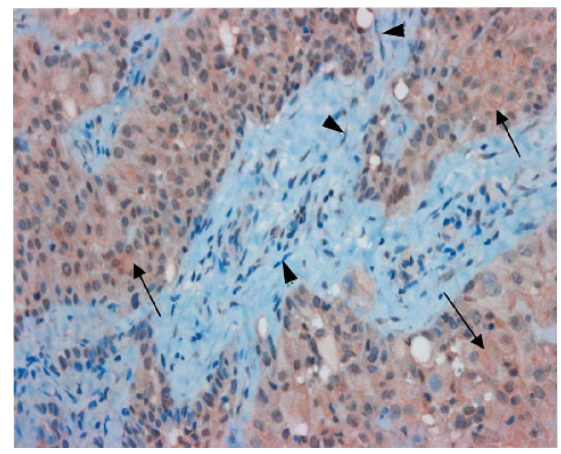

C

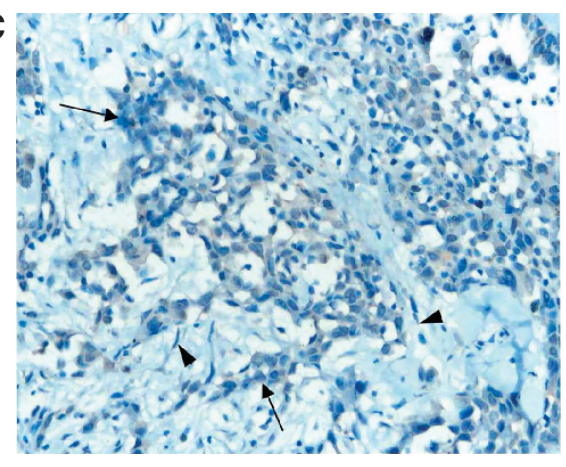

D

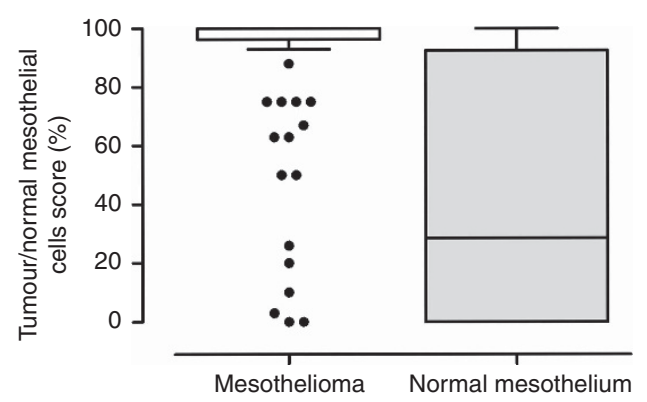

E

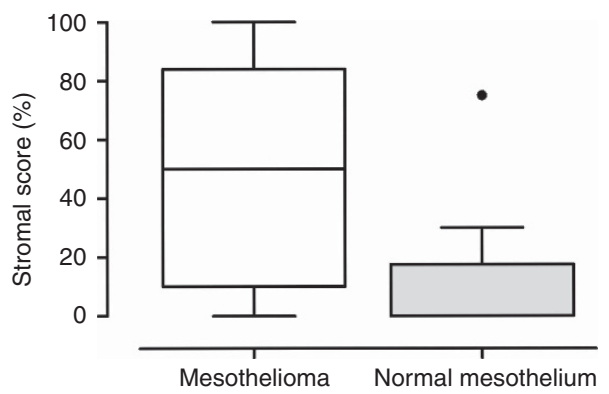

$\mathbf{F}$

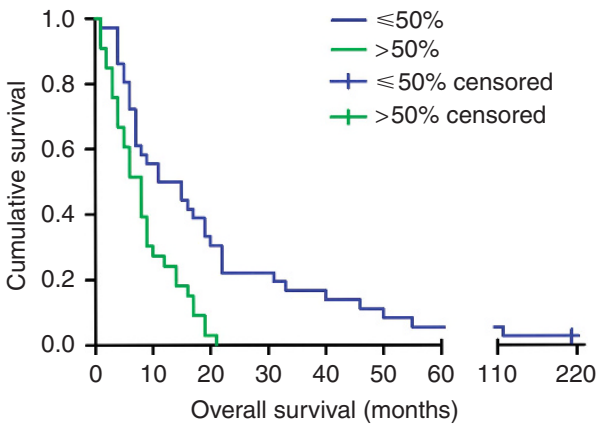

Figure 2. Immunohistochemical staining for SPARC. (A) Sarcomatoid histological subtype with high SPARC expression in tumour cells (arrows) and stroma (arrowheads). (B) Epithelioid mesothelioma with high SPARC expression in tumour (arrows) and low SPARC expression in stromal cells (arrowheads). (C) Epithelioid mesothelioma with low SPARC expression in tumour (arrows) and low SPARC expression in stromal cells (arrowheads). SPARC immunohistochemistry scores for mesothelioma compared with normal pleural mesothelium are shown in (D) tumour cells and (E) stromal fibroblasts. Kaplan-Meier curve for patients undergoing P/D according to stromal SPARC is shown in (F).

Table 3. Association of SPARC protein expression and patient characteristics in the P/D patients (series three)

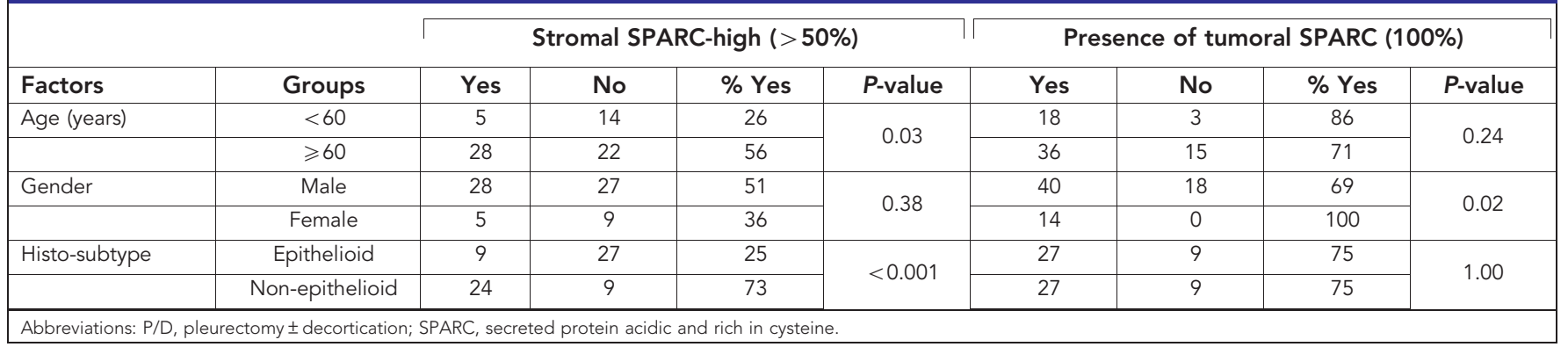

Circulating SPARC is likely secreted from the stroma of MPM tumours. To investigate whether tumour or stromal cells are the source of circulating SPARC, we evaluated cellular and secreted SPARC levels in vitro. We found that cellular SPARC expression was higher in 5 out of 7 cell lines than in the two immortalised mesothelial lines (Figure 3). An association between histological subtype and cellular SPARC was not evident. Assessment of SPARC levels in cell-conditioned medium revealed a lack of secreted SPARC in all cell lines (Figure 3). This finding was mirrored by the observation that human SPARC was not detectable in the plasma or serum of MPM xenograft-bearing mice (Figure 3). In contrast, in a parallel study using the same samples, we were able to detect another secreted protein, Fibulin-3, in the cellconditioned medium of all cell lines and in the plasma of xenograft-bearing mice (Kirschner et al, 2015). The lack of secretion of SPARC by MPM cell lines suggests stromal cells within the tumour, rather than the tumour cells themselves, are the source of circulating SPARC. 


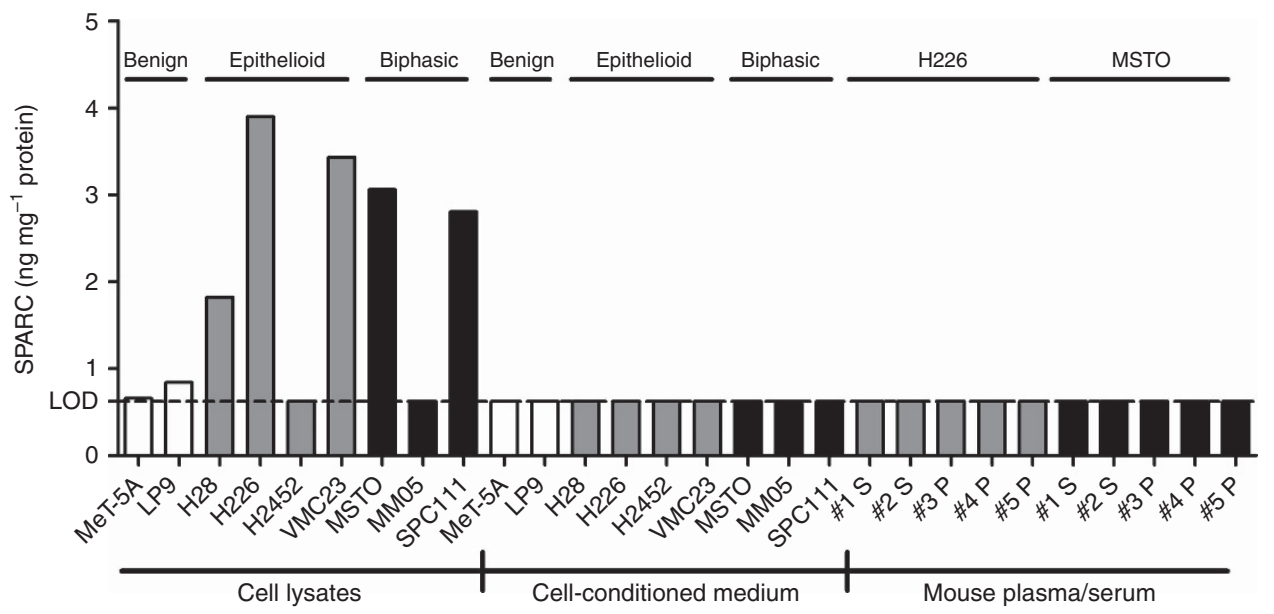

Figure 3. SPARC concentration measured by ELISA (ng per mg total protein) in cell lysates, cell-conditioned medium and mouse plasma/serum.

\section{DISCUSSION}

Our initial exploratory study demonstrated the feasibility of using iTRAQ proteomic techniques to investigate potential prognostic protein biomarkers in plasma of MPM patients. With this methodology, we have shown that it is possible to identify candidate plasma proteins with potential clinical significance, even in a relatively rare disease such as MPM. We used iTRAQ initially as a profiling tool to determine whether there were differentially expressed proteins between the two groups of 12 patients divided by their survival. We observed a good separation between the samples from long and short survivors when restricting our analysis to the five proteins identified as differentially expressed: SPARC, selenoprotein P, tetranectin, IBP2 and CXCL7. We then proceeded to quantify the selected protein levels by ELISA, which is often considered as the gold standard in protein quantification. We found high plasma levels of SPARC remained associated with short survival. This finding was consistent in the iTRAQ and ELISA experiments involving the 12 plasma samples of MPM patients, thus making this protein the most promising candidate biomarker in our exploratory study. To confirm this hypothesis-generating finding from our Australian patient cohort, we assessed serum SPARC concentrations using ELISA in an independent cohort of MPM patients from the Netherlands. We confirmed the prognostic value of circulating SPARC, adjusting for known prognostic factors such as age, gender, stage and histological subtypes.

While promising, our study is limited by its retrospective nature and the non-randomised allocation of treatment groups. Nevertheless, SPARC emerged as the most robust prognostic biomarker, given that the direction of prognostication was consistent between iTRAQ and ELISA in the discovery series as well as the demonstration of the independent prognostic value in the validation series. Further independent validation, preferably in a prospective study, is needed to confirm the prognostic value of circulating SPARC in MPM patients. A particular strength of our study is that it adheres to the REMARK guidelines where applicable, and uses pre-specified methods and analyses. Although a retrospective study, the characteristics of the study patients, the treatments received and the type of biological materials used and method of preservation and storage are clearly described. Most importantly, the multivariate model examining the independent significance of circulating SPARC in the Dutch series (our primary analysis) was fit to variables significant in the univariate analysis (SPARC and gender) as well as commonly accepted standard prognostic factors (age, histological subtype and stage), consistent with item 17 of the REMARK guidelines (McShane et al, 2005).
SPARC belongs to a group of non-structural components of the extracellular matrix that modulate interactions between cells and their environment (Tai and Tang, 2008), and is considered to be solely produced by the cancer-associated fibroblasts within tumours (Chlenski et al, 2006). Secreted protein acidic and rich in cysteine is known to be involved in regulating cell adhesion, proliferation, migration and tissue modelling; and it interferes with the binding of growth factors to their receptors in endothelial cells, resulting in inhibited proliferation (Hasselaar and Sage, 1992; Paulsson and Micke, 2014). Although SPARC expression is altered in many solid tumours, its role in tumorigenesis appears to be celltype specific due to the diverse functions of SPARC within the microenvironment of a given tumour (Chlenski and Cohn, 2010). In some types of cancer, such as melanoma, glioma, colorectal, osteosarcoma and gastric cancer, increased SPARC expression has been shown to be associated with disease progression and poor prognosis (Tai and Tang, 2008; Wang et al, 2014). which is consistent with our findings in MPM. In others, such as ovarian cancer, SPARC functions as a tumour suppressor with high levels correlating with good prognosis (Tai and Tang, 2008). However, there are inconsistent findings in the literature even within the same tumour type. For example, in non-small cell lung cancer, total SPARC expression (combined stromal and tumoral staining) correlated with unfavourable patient outcome (Koukourakis et al, 2003), while another study showed stromal SPARC expression to be associated with long patient survival (Huang et al, 2012), and a third study found no prognostic impact of stromal SPARC expression at all (Edlund et al, 2012).

Previous studies investigating the role of SPARC in cancer have examined the SPARC expression in tumour, either by IHC or by RT-qPCR, given its known biological function. As far as we know, this is the first report in the literature to demonstrate the prognostic importance of circulating SPARC in MPM patients. Given the detectable levels of circulating SPARC and demonstration of its prognostic significance, we hypothesised that the source of the circulating SPARC in our patients is the tumour itself. To that end, we showed that SPARC is present in both the tumour cells themselves and in the stromal components of the tumour with variable staining in the fibroblasts. Our demonstration that SPARC is expressed in both the tumour cells and stromal compartment is consistent with the expression of SPARC in both the epithelium and stroma of the prostate (Orr et al, 2012). Furthermore, we observed increased SPARC expression in MPM compared with normal mesothelial controls, suggesting a role for SPARC in the pathogenesis of MPM.

Interestingly, we found abundant SPARC in the lysates of most of the MPM cell lines, but none was secreted into the medium. This 
finding is consistent with a previous study, which showed that although both pancreatic cancer-derived fibroblasts and pancreatic cancer cell lines expressed SPARC, only the fibroblasts secreted SPARC in large quantities (Sato et al, 2003). One explanation for this observation could be related to the recent finding that the intracellular acidification frequently observed in cancer cells was able to reduce the secretion of SPARC and other extracellular matrix proteins by melanoma cells (Bellenghi et al, 2015). As human SPARC was not detectable in the serum or plasma of MPM xenograft-bearing mice, it further argues for a stromal origin for the SPARC detected in patient serum. Nevertheless, although our in vitro and in vivo experiments suggest that circulating SPARC is secreted from the stromal fibroblasts rather than the tumour cells, other cell types could be the source of SPARC, such as tumour-associated macrophages (Sangaletti et al, 2008). Further studies are required to definitively identify the origin of SPARC in patient serum.

In our current study, we did not examine the mechanism of SPARC upregulation in MPM compared with normal mesothelium. In glioma, miR-145 is downregulated, leading to increased expression of connective tissue growth factor (CTGF), which in turn leads to upregulation of SPARC (Lee et al, 2013). In MPM, it has been reported that CTGF is upregulated in rat sarcomatoid MPM (Jiang et al, 2014), and miR-145 is lower in MPM tumours and cell lines compared with normal tissues (Cioce et al, 2014). Therefore, we hypothesise that decreased miR-145 in MPM could in turn lead to increased CTGF and upregulated SPARC.

Examination of the functional role of SPARC in MPM was also beyond the scope of the current study. However, in gastric cancer where in line with our findings, there is a high SPARC expression in tumour and its expression is associated with poor prognosis, preclinical work has demonstrated that downregulation of SPARC inhibits invasion and growth of human gastric cancer cells (Yin et al, 2010). This is the subject of further study in our group. Prognostication in MPM is important given the heterogeneity of the tumour biology. If accurate prognostic stratification indicates a short time to death, then either palliative therapy or best supportive care alone may be appropriate; while a more aggressive approach to therapy may be offered if long survival is indicated. In addition to being a prognostic biomarker, SPARC may also be a potential predictive biomarker for chemotherapy sensitivity. There is an emerging association between tumoral and stromal SPARC and pancreatic adenocarcinoma (Neuzillet et al, 2013). Secreted protein acidic and rich in cysteine is overexpressed in the stroma of pancreatic cancer and has been investigated as a target for nabpaclitaxel. Nab-paclitaxel is a solvent-free, albumin-bound form of paclitaxel, which is an anti-microtubule agent used to treat a number of cancers. It has been shown that SPARC acts as a high-affinity receptor for albumin, resulting in increased intratumoral uptake of the drug. In a phase I/II study evaluating gemcitabine plus nabpaclitaxel in pancreatic adenocarcinoma, stromal SPARC expression was associated with improved OS, indicating that it may be a useful predictive biomarker for response to nab-paclitaxel-based regimen (Von Hoff et al, 2011). In a retrospective review of the SPARC expression in patients from the CONKO-001 study (randomised phase III study investigating the role of adjuvant gemcitabine $v s$ observation in resected pancreatic cancer), authors found that the negative prognostic impact was only restricted to patients who received adjuvant gemcitabine. This would suggest that SPARC is a predictive marker for response to gemcitabine, which is a chemotherapeutic agent commonly used in MPM (Sinn et al, 2014). Therefore, we believe that further clinical studies examining the predictive value of stromal SPARC expression in MPM patients undergoing gemcitabine or nab-paclitaxel would be worthwhile.

In conclusion, we have demonstrated that it is feasible to use well-validated proteomic methods to explore potential biomarkers in the plasma of MPM patients and showed SPARC to be a promising circulating prognostic biomarker. Furthermore, we validated the independent prognostic importance of circulating SPARC in a large retrospective series of MPM patients. While promising, this finding should be confirmed in a prospective manner before it could potentially be used clinically. We also demonstrated the presence of SPARC protein in the MPM tumour cells as well as the stromal fibroblasts, with our results indicating the stroma as the likely source of the circulating SPARC. We believe that our findings indicate the potential biological importance of SPARC in MPM and further studies examining the function of SPARC in MPM are warranted.

\section{ACKNOWLEDGEMENTS}

This work was funded by the Cancer Institute New South Wales (CINSW) Innovation Grant (to SC and NvZ) and the Workers' Compensation Dust Diseases Board (DDB) of New South Wales Research Grant Scheme (to GR, SCK, MM, SC and NvZ). Part of this work was undertaken at APAF, with the infrastructure provided by the Australian Government through the National Collaborative Research Infrastructure Strategy (NCRIS). Support from the National Foundation for Medical Research and Innovation is also gratefully acknowledged for part of this study.

\section{CONFLICT OF INTEREST}

The authors declare no conflict of interest.

\section{REFERENCES}

Bellenghi M, Puglisi R, Pedini F, De Feo A, Felicetti F, Bottero L, Sangaletti S, Errico MC, Petrini M, Gesumundo C, Denaro M, Felli N, Pasquini L, Tripodo C, Colombo MP, Care A, Mattia G (2015) SCD5-induced oleic acid production reduces malanoma malignancy by intracellular retention of SPARC and cathepsin B. J Pathol 236(3): 315-325.

Cerciello F, Choi M, Nicastri A, Bausch-Fluck D, Ziegler A, Vitek O, FelleyBosco E, Stahel R, Aebersold R, Wollscheid B (2013) Identification of a seven glycopeptide signature for malignant pleural mesothelioma in human serum by selected reaction monitoring. Clin Proteomics 10(1): 16 .

Chlenski A, Cohn S (2010) Modulation of matrix remodeling by SPARC in neoplastic progression. Semin Cell Dev Biol 21(1): 55-65.

Chlenski A, Liu S, Guerrero L, Yang Q, Tian Y, Salwen H, Zage P, Cohn S (2006) SPARC expression is associated with impaired tumor growth, inhibited angiogenesis and changes in the extracellular matrix. Int $J$ Cancer 118(2): 310-316.

Cioce M, Ganci F, Canu V, Sacconi A, Mori F, Canino C, Korita E, Casini B, Alessandrini G, Cambria A, Carosi M, Blandino R, Panebianco V, Facciolo F, Visca P, Volinia S, Muti P, Strano S, Croce C, Pass H, Blandino G (2014) Protumorigenic effects of mir-145 loss in malignant pleural mesothelioma. Oncogene 33(46): 5319-5331.

Edlund K, Lindskog C, Saito A, Berglund A, Ponten F, Goransson-Kultima H, Isaksson A, Jirstrom K, Planck M, Johansson L, Lambe M, Homberg L, Nyberg F, Ekman S, Berggvist M, Landelius P, Lamberg K, Botling J, Ostman A, Micke P (2012) CD99 is a novel prognostic stromal marker in non-small cell lung cancer. Int J Cancer 131(10): 2264-2273.

Gentleman RC, Carey VJ, Bates DM, Bolstad B, Dettling M, Dudoit S, Ellis B, Gautier L, Ge Y, Gentry J, Hornik K, Hothorn T, Huber W, Iacus S, Irizarry R, Leisch F, Li C, Maechler M, Rossini AJ, Sawitzki G, Smith C, Smyth G, Tierney L, Yang LY, Zhang J (2004) Bioconductor: open software development for computational biopsy and bioinformatics. Genome Biol 5(10): R80.

Grigoriu B-D, Scherpereel A, Devos P, Chahine B, Letourneux M, Lebailly P, Gregoire M, Porte H, Copin M-C, Lassalle P (2007) Utility of osteopontin and serum mesothelin in malignant pleural mesothelioma diagnosis and prognosis assessment. Clin Cancer Res 13(10): 2928-2935.

Hasselaar P, Sage EH (1992) SPARC antagonizes the effect of basic fibroblast growth factor on the migration of bovine aortic endothelial cells. J Cell Biochem 49(3): 272-283. 
Huang Y, Zhang J, Zhao Y, Jiang W, Xue C, Xu F, Zhao H, Zhang Y, Zhao L, Hu Z, Yao Z, Liu Q, Zhang L (2012) SPARC expression and prognostic value in non-small cell lung cancer. Chin J Cancer 31(11): 541-548.

Jankova L, Chan C, Fung CL, Song X, Kwun SY, Cowley MJ, Kaplan W, Dent OF, Bokey EL, Chapuis PH, Baker MS, Robertson GR, Clarke SJ, Molloy MP (2011) Proteomic comparison of colorectal tumours and nonneoplastic mucosa from paired patient samples using iTRAQ mass spectrometry. Mol Biosyst 7(11): 2997-3005.

Jiang L, Yamashita Y, Chew S, Akatsuka S, Ukai S, Wang S, Nagai H, Okazaki Y, Takahashi T, Toyokuni S (2014) Connective tissue growth factor and $\beta$-catenin constitute an autocrine loop for activation in rat sarcomatoid mesothelioma. J Pathol 233(4): 402-414.

Kao S, Lee K, Armstrong N, Clarke S, Vardy J, Van Zandwijk N, Reid G, Burn J, McCaughan B, Henderson D, Klebe S (2011) Validation of tissue microarray technology in malignant pleural mesotheloma. Pathology 43(4): 128-132.

Kao S, Harvie R, Paturi F, Taylor R, Davey R, Abraham R, Clarke S, Marx G, Cullen M, Kerestes Z, Pavlakis N (2012) The predictive role of serum VEGF in an advanced malignant mesothelioma patient cohort treated with thalidomide alone orcombined with cisplatin/gemcitabine. Lung Cancer 75(2): 248-254.

Kirschner M, Pulford E, Mir Hoda A, Rozsas A, Griggs K, Cheng Y, Edelman J, Kao S, Hyland R, Dong Y, Laszlo V, Klikovits T, Vallely M, Grusch M, Hegedus B, Dome B, Klepetko W, Van Zandwijk N, Klebe S, Reid G (2015) Fibulin-3 has a potential prognostic, but not diagnostic role in malignant pleural mesothelioma. Br J Cancer 113(6): 963-969.

Koukourakis M, Giatromanolaki A, Brekken R, Sivridis E, Gatter K, Harris A, Sage E (2003) Enhanced expression of SPARC/osteonectin in the tumor-associated stroma of non-small cell lung cancer is correlated with markers of hypoxia/ acidity and with poor prognosis of patients. Cancer Res 63(17): 5376-5380.

Kryeziu K, Jungwirth U, Hoda M, Ferk F, Knasmuller S, Karnthaler-Benbakka C, Kowol C, Berger W, Heffeter P (2013) Synergistic anticancer activity of arsenic trioxide with erlotiib is based on inhibition of EGFR-mediated DNA double-strand break repair. Mol Cancer Ther 12(6): 1073-1084.

Lee H, Bier A, Cazacu S, Finniss S, Xiang C, Twito H, Poisson L, Mikkelsen T, Slavin S, Jacoby E, Yalon M, Toren A, Rempel S, Brodie C (2013) MicroRNA145 is downregulated in glial tumors and regulates glioma cell migration by targeting connective tissue growth factor. PLoS One 8(2): e54652.

Linton A, Cheng Y, Griggs K, Kirschner M, Gattani S, Srikaran S, Kao S, McCaughan B, Klebe S, Van Zandwijk N, Reid G (2014) An RNAi-based screen reveals PKL1, CDK1 and NDC80 as potential therapeutic targets in malignant pleural mesothelioma. Br J Cancer 110(2): 510-519.

McShane LM, Altman DG, Sauerbrei W, Taube SE, Gion M, Clark GM. Statistics Subcommittee of the NCI-EORTC Working Group on Cancer Diagnostics (2005) Reporting recommendations for tumor marker prognostic studies. J Clin Oncol 23(36): 9067-9072.

Neuzillet C, Tijeras-Raballand A, Cros J, Faivre S, Hammel P, Raymond E (2013) Stromal expression of SPARC in pancreatic adenocarcinoma. Cancer Metastasis Rev 32(3-4): 585-602.

Orr B, Riddick A, Stewart G, Anderson R, Franco O, Hayward S, Thomson A (2012) Identification of stromally expressed molecules in the prostate by tag-profiling of cancer-associated fibroblasts, normal fibroblasts and fetal prostate. Oncogene 31(9): 1130-1142.

Ostroff R, Mehan M, Stewart A, Ayers D, Brody E, Williams S, Levin S, Black B, Harbut M, Carbone M, Goparaju C, Pass H (2012) Early detection of malignant pleural mesothelioma in asbestos-exposed individuals with a noninvasive proteomics-based surveillance tool. PLoS One 7(10): e46091.

Pass H, Levin S, Harbut M, Melamed J, Chirboga L, Donington J, Huflejt M, Carbone M, Chia D, Goodglick L, Goodman G, Thornquist M, Liu G, De Perrot M, Tsao M, Goparaju C (2012) Fibulin-3 as a blood and effusion biomarker for pleural mesothelioma. N Engl J Med 367(15): 1417-1427.

Paulsson J, Micke P (2014) Prognostic relevance of cancer-associated fibroblasts in human cancer. Semin Cancer Biol 25: 61-68.

Relan V, Morrison L, Parsonson K, Clarke B, Duhig E, Windsor M, Mater K, Naidoo R, Passmore L, Mccaul E, Courtney D, Yang I, Fong K, Bowman R (2013) Phenotypes and karyotypes of human malignant mesothelioma cell lines. PLoS One 8(3): e58132.

Roe O, Creaney J, Lundgren S, Larsson E, Sandeck H, Boffetta P, Nilsen T, Robinson BWS, Kjaerheim K (2008) Mesothelin-related predictive and prognostic factors in malignant mesothelioma: a nested case-control study. Lung Cancer 61(2): 235-243.

Ross P, Huang Y, Marchese J, Williamson B, Parker K, Hattan S, Khainovski N, Pillai S, Dey S, Daniels S, Purkayastha S, Juhasz P, Martin S, BartletJones M, He F, Jacobson A, Pappin D (2004) Multiplexed protein quantitation in Saccharomyces cerevisiae using amine-reactive isobaric tagging reagents. Mol Cell Proteomics 3(12): 1154-1169.

Sangaletti S, Di Carlo E, Gariboldi S, Miotti S, Cappetti B, Parenza M, Rumio C, Brekken RA, Chiodoni C, Colombo MP (2008) Macrophage-derived SPARC bridges tumor cell-extraceullar matrix interactions toward metastasis. Cancer Res 68(21): 9050-9059.

Sato N, Fukushima N, Maehara N, Matsubayashi H, Koopmann J, Su GH, Hruban RH, Goggins M (2003) SPARC/osteonectin is a frequent target for aberrant methylation in pancreatic adenocarcinoma and a mediator of tumor-stromal interactions. Oncogene 22(32): 5021-5030.

Scherpereel A, Astoul P, Baas P, Berghmans T, Clayson H, De Vuyst P, Dienemann H, Galateau-Salle F, Hennequin C, Hillerdal G, Le Pechoux C, Mutti L, Pairon J-C, Stahel R, Van Houtte P, Van Meerbeeck J, Waller D, Weder W (2010) Guidelines of the European Respiratory Society and the European Society of Thoracic Surgeons for management of Malignant Pleural Mesothelioma. Eur Respir J 35(3): 479-495.

Schmitter D, Lauber B, Fagg B, Stahel R (1992) Hematopoietic growth factors secreted by seven human pleural mesothelioma cell lines: interleukin- 6 production as a common feature. Int J Cancer 51(2): 296-301.

Schneider J, Hoffmann H, Dienemann H, Herth F, Meister M, Muley T (2008) Diagnostic and prognostic value of soluble mesothelin-related proteins in patients with malignant pleural mesothelioma in comparison with benign asbestosis and lung cancer. J Thorac Oncol 3(12): 1317-1324.

Sinn M, Sinn B, Striefler J, Lindner J, Stieler J, Lohneis P, Bischoff S, Blaker H, Pelzer U, Bahra M, Dietel M, Dorken B, Oettle H, Riess H, Denkert C (2014) SPARC expression in resected pancreatic cancer patients treated with gemcitabine: results from the CONKO-001 study. Ann Oncol 25(5): $1025-1032$.

Song X, Bandow J, Sherman J, Baker JD, Brown PW, McDowell MT et al. (2008) iTRAQ experimental design for plasma biomarker discovery. J Proteome Res 7(7): 2952-2958.

Tai I, Tang M (2008) SPARC in cancer biology: its role in cancer progression and potential for therapy. Drug Resist Updat 11(6): 231-246.

Van Meerbeeck J, Scherpereel A, Surmont V, Baas P (2011) Malignant pleural mesothelioma: the standard of care and challenges for future management. Crit Rev Oncol Hematol 78(2): 92-111.

Vogelzang NJ (2008) Chemotherapy for malignant pleural mesothelioma. The Lancet 371(9625): 1640-1642.

Vogelzang NJ, Rusthoven JJ, Symanowski J, Denham C, Kaukel E, Ruffie P, Gatzemeier U, Boyer M, Emri S, Manegold C, Niyikiza C, Paoletti P (2003) Phase III study of pemetrexed in combination with cisplatin versus cisplatin alone in patients with malignant pleural mesothelioma. J Clin Oncol 21(14): 2636-2644.

Von Hoff D, Ramanathan R, Borad M, Laheru D, Smith L, Wood T, Korn R, Desai N, Trieu V, Iglesias J, Zhang H, Soon-Shiong P, Shi T, Rajeshkumar N, Maitra A, Hidalgo M (2011) Gemcitabine plus nab-paclitaxel is an active regimen in patients with advanced pancreatic cancer: a phase I/II trial. J Clin Oncol 29(34): 4548-4554.

Wang Z, Hao B, Yang Y, Wang R, Li Y, Wu Q (2014) Prognostic role of SPARC expression in gastric cancer: a meta-analysis. Arch Med Sci 10(5): 863-869.

Wu Y, Parker L, Binder N, Beckett M, Sinard J, Griffiths C, Rheinwald J (1982) The mesothelial keratins: a new family of cytoskeletal proteins identified in cultured mesothelial cells and nonkeratinizing epithelia. Cell 31(3 Pt 2): 693-703.

Yin J, Chen G, Liu Y, Liu S, Wang P, Wan Y, Wang X, Zhu J, Gao H (2010) Downregulation of SPARC expression decreases gastric cancer cellular invasion and survival. J Exp Clin Cancer Res 29: 59.

This work is published under the standard license to publish agreement. After 12 months the work will become freely available and the license terms will switch to a Creative Commons AttributionNonCommercial-Share Alike 4.0 Unported License. 